\title{
Chapter 9 \\ Contemporary Opportunities \\ in Nonsurgical Management \\ of Locoregionally Advanced Head \\ and Neck Squamous Cell Carcinoma
}

\author{
Shao Hui Huang, Avinash Pilar, Jishi Li, Zhiyuan Xu, and Brian O’Sullivan
}

\section{Introduction}

Mucosal head and neck squamous cell carcinoma (HNSCC) generally refers to carcinoma arising from the mucosa of the oro-/hypo-pharynx (excluding nasopharynx), larynx, oral cavity, and carcinoma of unknown primary origin presenting with cervical lymph node metastasis (CUP). Over the past decade, the landscape of HNSCC has changed dramatically owing to the rapid emergence of HPV-mediated

\footnotetext{
S. H. Huang

Department of Radiation Oncology, The Princess Margaret Cancer Centre, University of Toronto, Toronto, ON, Canada

Department of Otolaryngology-Head and Neck Surgery, The Princess Margaret Cancer Centre, University of Toronto, Toronto, ON, Canada

e-mail: shaohui.huang@rmp.uhn.ca
}

\author{
A. Pilar \\ Department of Radiation Oncology, The Princess Margaret Cancer Centre, University of \\ Toronto, Toronto, ON, Canada \\ e-mail: avinash.pilar@ rmp.uhn.ca \\ J. Li $\cdot \mathrm{Z} . \mathrm{Xu}$ \\ Department of Clinical Oncology, The University of Hong Kong - Shenzhen Hospital, \\ Shenzhen, China \\ e-mail: lijs@hku-szh.org; xuzy@hku-szh.org \\ B. O'Sullivan $(\triangle)$ \\ Department of Radiation Oncology, The Princess Margaret Cancer Centre, University of \\ Toronto, Toronto, ON, Canada \\ Department of Otolaryngology-Head and Neck Surgery, The Princess Margaret Cancer \\ Centre, University of Toronto, Toronto, ON, Canada \\ Department of Clinical Oncology, The University of Hong Kong - Shenzhen Hospital, \\ Shenzhen, China \\ e-mail: brian.osullivan@rmp.uhn.ca
}


$[\mathrm{HPV}(+)]$ oropharyngeal carcinoma (OPC) and a steady decrease in smokingrelated/HPV-negative $[\mathrm{HPV}(-)]$ HNSCC, the latter almost certainly explained by the success of smoking cessation strategies. The 8th edition TNM (TNM-8) now separates HNSCC into two major categories: $\operatorname{HPV}(+)$ and $\operatorname{HPV}(-) \operatorname{HNSCC}[1,2]$ with different staging classifications. Examples introduced in the TNM-8 include the migration of almost $50 \%$ of HPV(+) oropharyngeal cancer (OPC) from Stage IV in the traditional 7th edition TNM to Stage I in the TNM-8, the important introduction of depth of invasion in oral cancer that influences migration to more advanced T-categories, and the assignment of node-positive $(\mathrm{N}+)$ disease with extranodal nodal extension (ENE) to higher $\mathrm{N}$ categories in $\mathrm{HPV}(-)$ disease. As a consequence, the semantics of "locoregionally advanced HNSCC" (LAHNSCC) is also evolving.

Achieving locoregional control (LRC) has traditionally been the primary focus of management of LAHNSCC due to the challenge in achieving it with conventional treatment approaches in use in the pre-HPV era. As well, recurrence in this location almost uniformly has significant implications for symptomatology, function, severe morbidity (involving the integrity of airway, neurovascular, and musculoskeletal structure), swallowing, and nutrition in addition to the hardships and risks associated with salvage management. Hence, a long-established sentiment prevailed that LAHNSCC was a "loco-regional disease" and less effort was devoted to negating the risk of distant metastasis (DM). Concurrent cisplatin-based chemoradiotherapy (CCRT) has represented the gold standard for organ preservation treatment in LAHNSCC since the publication of the MACH-NC meta-analysis which demonstrated significant improvement in LRC and OS with the addition of chemotherapy to radiotherapy $(\mathrm{RT})[3,4]$. Despite this, the LRC rates remain unsatisfactory for many HPV(-) LAHNSCC. About $40 \%$ of patients experience locoregional failure (LRF) [5] and less than 50\% of HPV(-) LAHNSCC patients survive more than 5 years [6]. Efforts have continued to explore other systematic approaches to enhance $\mathrm{LRC}$ in this population.

As is the case for HPV(-) LAHNSCC, HPV(+) OPC is also facing challenges, although of a different nature. While LRC can be achieved in $>80 \% \mathrm{HPV}(+)$ OPCs [5-8], most of these patients received intensified treatment and can expect to live for many years but are vulnerable to severe late toxicities that significantly affect quality of life in many cancer survivors. In addition, DM has emerged as one of the major challenges for this population and approaches confronting this outcome are relatively sparse. To improve the therapeutic ratio of HPV(+) LAHNSCC, the current overall research focus in this population has shifted towards two scenarios: safe de-intensification for the low relapse risk group, while innovative approaches to improve LRC and mitigate the risk of DM remain priorities in the high relapse risk group.

Non-surgical approaches for HNSCC have also evolved in parallel with accumulating knowledge about disease biology and clinical behavior, advances in technologies, availability of novel treatment approaches, and emerging evidence from clinical trials and prospective/retrospective studies. While surgery remains a mainstay in management to ensure local control, and radical RT with/without chemotherapy is similarly hallowed in the overall management philosophy, changes in 
approach for different presentations are under evaluation. In this review, we summarize recent research findings in non-surgical approaches for both $\mathrm{HPV}(+)$ and HPV(-) LAHNSCC, including revisiting the efficacy of traditional chemotherapy agents, the role of epidermal growth factor receptor (EGFR) inhibition, the potential to refine chemotherapy regimens (including new agents and sequencing), and the combination of immunotherapy with RT.

\section{Definition of LAHNSCC in HPV(+) HNC}

LAHNSCC has historically referred to stage III/IV disease that included T3-T4 or any N-positive presentations. In the HPV(+) TNM-8 classification, the TNM-7 T1-T2_N1-N2b subset has been re-classified as stage I disease with very high LRC and low DM risk [5]. Since no stage IV group exists for nonmetastatic HPV(+) OPC/CUP, stage II and III would naturally be considered as LAHNSCC. However compelling data indicates that outcome heterogeneity still exists within stage I. Stage I disease with radiologically identified extranodal extension (rENE+) has emerged as a strong prognostic factor for higher DM and mortality risk [9], and a proposal exists to classify it as N3, and therefore stage III disease [10]. By extension it seems prudent to also combine HPV(+) TNM-8 stage I disease with rENE+, together with stage II/III, as LAHNSCC group.

The nominal components of the main risk strata for HPV(-) LAHNCC have not changed in TNM-8 and continue to refer to stage III and IV disease, including all non-metastatic (M0) HPV(-) LAHNSCC excepting the T1-T2 N0 subset. However, the criteria contributing to individual $\mathrm{T}$ and $\mathrm{N}$ categories have been refined which has resulted in criterion-based stage modification. A depth of invasion (DOI) parameter has been added as a new T-category modifier for oral cavity SCC and migrates so called "thicker" tumours (correspondingly those with higher DOI) to a more advanced T-category. Clinical and pathological descriptors for ENE have also been introduced that assign a higher N-category. Such changes in definitions warrant reinterpretation of historical data and impact present and future clinical trial design.

\section{Trials on HPV(+) LAHNSCC}

\section{Revisiting the Role of Cisplatin and Cetuximab in HPV(+) LAHNSCC}

With the recognition of $\mathrm{HPV}(+) \mathrm{HNSCC}$ as a new disease, clinical trials are addressing HPV(+) HNSCC separately from HPV(-) disease. The most established "tool" for LAHNSCC is cisplatin chemotherapy combined with RT. Cetuximab, an FDA approved EGFR inhibitor, has also been used in LAHNSCC following a randomized 
trial (IMCL-9815) that showed superior LRC with cetuximab combined with RT compared to RT alone for LAHNSCC; however HPV status was unknown at the time of the trial [11] and the RT outcomes may not reflect the results expected from contemporary precision RT techniques which were unavailable during the period of the trial. The efficacy and toxicity of cisplatin and cetuximab with RT on HPV(+) HNSCC were recently compared in the two $\mathrm{HPV}(+)$ phase-III randomized trials: RTOG 1016 (NCT01302834) [7] (comprising 39\% T3-T4 tumours) and De-ESCALaTE HPV (NCT01874171) [8] (comprising 34\% T3-T4 tumours). Both trials showed inferior efficacy of cetuximab compared to cisplatin in $\mathrm{HPV}(+) \mathrm{OPC}$, mainly attributable to higher LRF with cetuximab. The differential effect on DM reduction with cisplatin versus cetuximab was significant in De-ESCALaTE HPV (2-year DM: 3\% vs. 9\%, $\mathrm{p}=0.009$ ) but marginal in RTOG 1016 (5-year DM: 8.6\% vs. 11.7\%, p = 0.09). Regarding outcomes according to TNM-8, the De-ESCALaTE HPV trial showed that the differential effect of cisplatin vs. cetuximab exists in both stage I/II (98.4\% vs. $93.2 \%, \mathrm{p}=0.043$ ) and stage III diseases (2-year OS: $93.3 \%$ vs. $67.1 \%, \mathrm{p}=0.030$ ). The toxicity profile also did not favor cetuximab. The failure of cetuximab to optimize outcomes in the loco-regional treatment of $\mathrm{HPV}(+) \mathrm{OPC}$ is probably not surprising when one considers that HPV(+) OPC rarely expresses EGFR [12]. An additional intriguing observation of the RTOG 1016 trial is the relatively high LRF in the cetuximab arm compared to other reported outcomes with RT alone in $\mathrm{HPV}(+)$ cohorts $[13,14]$. Compromised outcomes of cetuximab in HPV(+) OPC was also observed in the RTOG 0522 trial (NCT00265941) [15]. It showed a trend towards higher hazard ratio (HR $1.57, \mathrm{p}=0.12$ ) with the addition of cetuximab to cisplatin chemotherapy which was opposite to that found with HPV(-) OPC (HR 0.86, p = 0.31). These paradoxical observations raise an unsubstantiated possibility for cetuximab to be interfering with radiosensitivity in the treatment of $\mathrm{HPV}(+)$ OPC.

Notwithstanding any additional nuances, both aforementioned phase III trials have cemented the place of cisplatin as a potent radiosensitizer to enhance LRC although it is less effective in abrogating the risk of DM. Cisplatin combined with RT remains the gold standard for the treatment of HPV(+) LAHNSCC while cetuximab is not suitable for this disease. Several important questions regarding chemotherapy remain unresolved. For example, there is no robust data to indicate which subgroups of patients truly benefit from cisplatin chemotherapy and there remains uncertainty about the optimal dose of cisplatin for $\mathrm{HPV}(+)$ OPC patients. A retrospective study suggests that a cumulative dose of cisplatin $>200 \mathrm{mg} / \mathrm{m}^{2}$ seems necessary for TNM-8 stage III (T4 or N3) HPV(+) OPC [16]. Another prominent question is whether weekly cisplatin is equally effective compared to three-weekly high dose cisplatin (a trial is currently under development). The NRG HN-002 trial (NCT02254278) showed that weekly cisplatin with reduced RT dose (60 Gy in 30 fractions, 5 fractions per week) is very effective for T1-T3N0-N2b HPV(+) OPC minimal smokers $(<10$ pack-year smoking) with 2-year progression free survival of $90.5 \%$ [17]. However, the trial shows that, while LRC is achievable with cisplatin combined with a modest RT dose reduction, cisplatin also appears to be less potent in fully mitigating DM risk. For example, the RTOG 0234 trial, although without knowledge of HPV status, showed that docetaxel in combination with cetuximab seemed more effective compared to cisplatin with cetuximab in DM reduction (2-year DM: $13 \%$ vs. $25 \%$ ) in the 
postoperative setting of general LAHNSCC [18]. In essence, more effective systemic agents are needed to eradicate microscopic metastasis overall and in $\mathrm{HPV}(+)$ LAHNSCC due to the prominence of this end-point in the management of the disease.

\section{Refining “Old Tools" for HPV(+) LAHNSCC: Dose, Fractionation, and Volumes}

Although most HPV(+) LAHNSCC have good outcomes, RT intensification is still needed for a subset of HPV(+)LAHNSCC. In addition to cisplatin radiosensitization, other traditional intensification "tools" include hyper-fractionation with augmented RT doses, shortened overall treatment time (acceleration) [19], or hypoxia modification (e.g. nimorazole combined with radiotherapy). Studies have shown that an acceleration using six fractions compared to five fractions per week improved the outcome of HPV(+) OPC [20]. The NRG HN-002 trial (NCT02254278) also indicated that even in "low-risk" minimal smoking N0-N1 HPV(+) OPSCC, modest dose intensification by fractionating $60 \mathrm{~Gy}$ in 30 fractions over 5 weeks ( 6 fractions per week) rather than 6 weeks for the treatment period may still be beneficial [17]. Hypoxia modification has not shown effectiveness in $\mathrm{HPV}(+) \mathrm{OPC}$ although it improves outcomes in HPV(-) LAHNSCC [21].

Another traditional "tool" under active study in $\mathrm{HPV}(+)$ OPC is refining the elective RT volumes. Villaflor et al. [22] conducted a phase II trial and showed that volume reduction (omitting the elective volume that ordinarily treats regions of the neck that are not overtly involved by disease) in patients with complete or partial response (amounting to at least a 50\% volume reduction) after induction chemotherapy appears to be safe. Patients in the subsequent OPTIMA trial [23] also received risk-stratified dose-volume reduction and de-escalated RT volumes which were limited to the first echelon of uninvolved nodes with promising results. Longterm follow-up of the trial patients with additional patients treated following OPTIMA outlines presented in ASCO 2020 confirmed safety and excellent functional outcomes with this approach [24]. The HN10 trial (NCT03822897) of the Canadian Clinical Trials Group (CCTG), a phase II single-arm trial of Elective Volume Adjusted De-Escalation Radiotherapy (EVADER) for TNM-8 stage I-II $\mathrm{HPV}(+)$ OPSCC is currently recruiting and adjusts the prophylactic RT neck volumes according to the initial sites of disease presentation (e.g. the presenting subsite in the oropharynx, laterality of the primary site, and the extent of neck node disease).

\section{Addressing Distant Metastasis Endpoint: Role of Induction Chemotherapy}

Induction chemotherapy has been proven to be effective in DM reduction in nasopharyngeal carcinoma [25, 26], another viral-related pharyngeal cancer. GP (gemcitabine-cisplatin) induction chemotherapy appears to have similar efficacy in 
DM reduction with lower grade 3-4 toxicities compared to the TPF (docetaxelcisplatin-fluorouracil) regimen. However, the role of induction chemotherapy in HPV(+) OPC is yet to be defined. The phase III DeCIDE trial (NCT00117572) [27] compared TPF induction chemotherapy followed by cisplatin-CCRT vs. cisplatinCCRT alone in N2-N3 HNSCC [61\% were OPC, of which the majority were $\mathrm{HPV}(+)$ ]. The induction chemotherapy cohort showed a significant reduction in DM as the first site of failure $(\mathrm{p}=0.043)$, but this difference did not translate into an OS difference. A possible reason is that the trial was based on the TNM-7 classification and many $\mathrm{N} 2 \mathrm{HPV}(+)$ OPC enrolled in the trial had traditional N2b disease with T1-T2 categories which today would be considered low risk by TNM-8. In turn this could have diluted a putative benefit of induction chemotherapy. Similarly, the phase III PARADIGM trial (NCT00095875) [28] investigated the role of TPF induction chemotherapy followed by carboplatin-CCRT vs. cisplatin-CCRT alone in LAHNSCC (tumour HPV status was not tested) and also did not find a survival benefit. The trial was terminated early due to slow accrual. More recently, the single-arm phase II ECOG 1308 trial [29] and the OPTIMA trial [23] both suggested a promising role for induction chemotherapy in DM risk reduction, as well as a risk stratification tool for refining subsequent treatment including, most importantly, the potential to administer a less intense locoregional approach in appropriately responding cases following the induction regimen.

\section{Quest for Additional Risk Stratification Parameters}

Although TNM-8 stratifies HPV(+) OPC patients' prognosis better than TNM-7, it is recognized that outcome heterogeneity exists, especially in stage I disease [9]. Recently, rENE+ was observed to carry strong prognostic value, mainly impacting DM. A resulting proposal considers that all cases with $\mathrm{rENE}+$ should be classified as $\mathrm{N} 3 \mathrm{~b}$ disease since it portends higher risk of DM and worse OS among all nonmetastatic (M0) HPV(+) OPC [10]. The study also found that the addition of cisplatin could negate the LRF risk with rENE+ but does not appreciably negate DM risk. Therefore, strategies addressing the DM endpoint are urgently needed. One of the challenges of implementing rENE+ as a risk stratification factor is how to reliably assess rENE+. For example, "conglomerate", "matted" nodes, and "coalescent" nodes could all indicate evidence of rENE+ in addition to irregular nodal borders and adjacent structure invasion [10]. Radiologist training and standardization of taxonomy is needed. Computer-assisted intelligent machine learning may also enhance sensitivity and objectivity in recognizing rENE+ $[30,31]$. Notably, the need to restrict the designation of rENE+ to only cases with obvious and unequivocal criteria is potentially important. "Overcall" of rENE+ by inclusion of cases where extranodal disease is either not actually present or of minimal degree could obscure the very deleterious true impact of unequivocal rENE+, especially on DM and mortality. Recent evidence suggests that the associated risk surpasses that of other accepted prognostic factors, including TNM stage and its categories, and smoking history. 
Besides rENE+, researchers are also investigating other biomarkers for risk stratification of HPV(+) LAHNSCC. Dynamic biomarker such as the pre-treatment tumour growth velocity [32], response to induction chemotherapy [29], or the temporal pace of morphological [33] and functional (by FDG PET or hypoxia imaging) [34] volume reduction during the early phase of the RT course are potential candidates for risk stratification and merit investigation with response-adapted clinical trials.

HPV genotyping may also have a potential role for risk stratification. High-risk HPV includes $\alpha-7$ HPV subtype (e.g. HPV-18, 39, 45) and $\alpha-9$ HPV subtype (e.g. HPV-16, 31, 33, 35) [35-37]. The majority (>95\%) of HPV(+) OPC is caused by HPV-16 followed by HPV-35 and HPV-31, and rarely by HPV-18 and HPV-45 [3841]. Emerging data suggest that patients with an $\alpha-7$ HPV subtype (e.g. HPV-18) OPC do not have as good prognosis as those caused by an $\alpha-9$ HPV subtype (e.g. HPV16, 31, 33, 35, etc.) [35-37]. Whether a patient with $\alpha-7$ HPV driven OPC should be excluded from de-intensification trials remains to be determined.

Liquid biopsy has shown a promising role in risk stratification as well. A recent report from Fakhry et al. [42] showed that oral HPV DNA viral load detected using oral rinse decreased rapidly with therapy, and persistent detection was associated with increased risk of recurrence and death. Analysis of tumour HPV DNA holds considerable promise as a biomarker for treatment response and risk of progression. Chera et al. [43] demonstrated the potential role of plasma circulating HPV DNA in disease surveillance.

\section{Emerging Role of Immunotherapy in HPV(+) LAHNSCC}

Emerging evidence suggests that the host immune system plays a significant role in the outcome of cancer patients. HPV(+) OPC is an immunogenic tumour [44, 45], rendering it a potential target tumour site for immunotherapy. A recent study revealed that a majority of $\mathrm{HPV}(+) \mathrm{OPC}$ had PD-L1 overexpression, especially those with a minimal smoking history (93\%), and was higher than in HPV(-) OPC $(70 \%)$ although the prognostic value of the finding remains uncertain [46].

Currently, available immunotherapy strategies include passive immunotherapy (e.g. immune checkpoints inhibitors, immune co-stimulatory antibodies, tumorinfiltrating lymphocytes, and chimeric antigen receptor [CAR] $\mathrm{T}$ cells) and active immunotherapy (e.g. vaccines, immune adjuvant cytokines, and oncolytic viruses) [47]. Thus far, immune checkpoint inhibition is the most commonly investigated immunotherapy strategies for HNSCC. Several strategies exist to block the intrinsic inhibitory immune checkpoint pathways. For example, programmed cell death protein (PD-1)/programmed death-ligand 1 (PD-L1) pathway blockade restores the activity of anti-tumour $\mathrm{T}$ cells that have become dormant while cytotoxic T-lymphocyte-associated antigen 4 (CTLA-4) blockade allows for activation and proliferation of more cytotoxic T-cell clones and reduces T-cell mediated immunosuppression. PD-1 blockade has shown promising results in the recurrent/metastatic 
setting [48-51], which prompted approval of nivolumab or pembrolizumab, both PD-1 inhibitors, by the Food and Drug Administration (FDA) for treatment of recurrent/metastatic HNSCC.

Theoretically, radiotherapy can be synergistic with immunotherapy to enhance its effect [52]. For example, RT may prime the immune system to release and/or expose tumour-specific antigens to elicit tumour-specific T cell responses $[52,53]$. Conversely, RT could also suppress the immune system when a high RT dose is delivered to large volumes of hematologic cells [54]. The balance of priming or suppressing the immune response may depend on RT dose, fraction size, delivery time, as well as the irradiated volume. Not surprisingly, the focus has shifted to novel approaches including investigation of the role of immunotherapy combined with RT in the definitive setting (Table 9.1). KEYNOTE 412 (NCT03040999), a phase III trial $(\mathrm{n}=780)$, examined the addition of pembrolizumab to CCRT compared to CCRT alone for LAHNSCC, including T4 or N3 HPV(+) OPC and p16-negative stage III/IV (except TNM-7 T1-T2N1) OPC and larynx/hypopharynx/oral cavity SCC. The trial has completed recruitment and results are awaited. The JAVELIN Head and Neck 100 trial (NCT02952586) $(n=697)$ [55] was designed to evaluate the addition of avelumab (a PD-L1 inhibitor) to CCRT for LAHNSCC including $\mathrm{HPV}(+)$ T4 or N2c-N3 (TNM-8 stage II/III) disease and stage III/IV HPV(-) LAHNSCC. However, an interim analysis of the trial results suggested a lack of efficacy leading to termination of accrual [56]. Nonetheless, such trials may be able to shed light on whether PD-L1 expression is a harbinger of adverse prognosis, while at the same time confer useful prediction by indicating a possible benefit of anti-PD-L1 immunotherapeutic agents.

Besides PD-1/PD-L1 inhibition (thereby blocking immune-suppressing ligands) that unleashes T-cell anti-tumour function, CTLA-4 blockage could enhance T-cell activation and is also under evaluation in HPV(+) OPC. Since PD-1/PD-L1 and CTLA-4 block different target pathways, it is hypothesized that targeting both PD-1/PD-L1 and CTLA-4 pathways may have additive or synergistic activity, although toxicity is unknown. One such trial is the CTTG HN.9 trial (NCT03410615) which was designed with the intent of comparing two arms containing RT with either concurrent-adjuvant durvalumab (PD-L1 inhibitor) versus durvalumab and tremelimumab (CTLA-4 inhibitor) compared to a third arm comprising standard of care cisplatin-CCRT. Several EORTC centers are also currently joining this trial. Notably, the tremelimumab arm has been terminated prematurely due to excessive adverse events.

\section{Research in HPV(-) LAHNSCC}

In contrast to the numerous trials and a variety of investigational approaches targeting $\mathrm{HPV}(+) \mathrm{HNSCC}$, the trial arena for HPV(-) HNSCC remains relatively quiet. The outcome of HPV(-) LAHNSCC with the current standard of care (high dose cisplatin-CCRT) remains unsatisfactory. Novel strategies are urgently needed for 


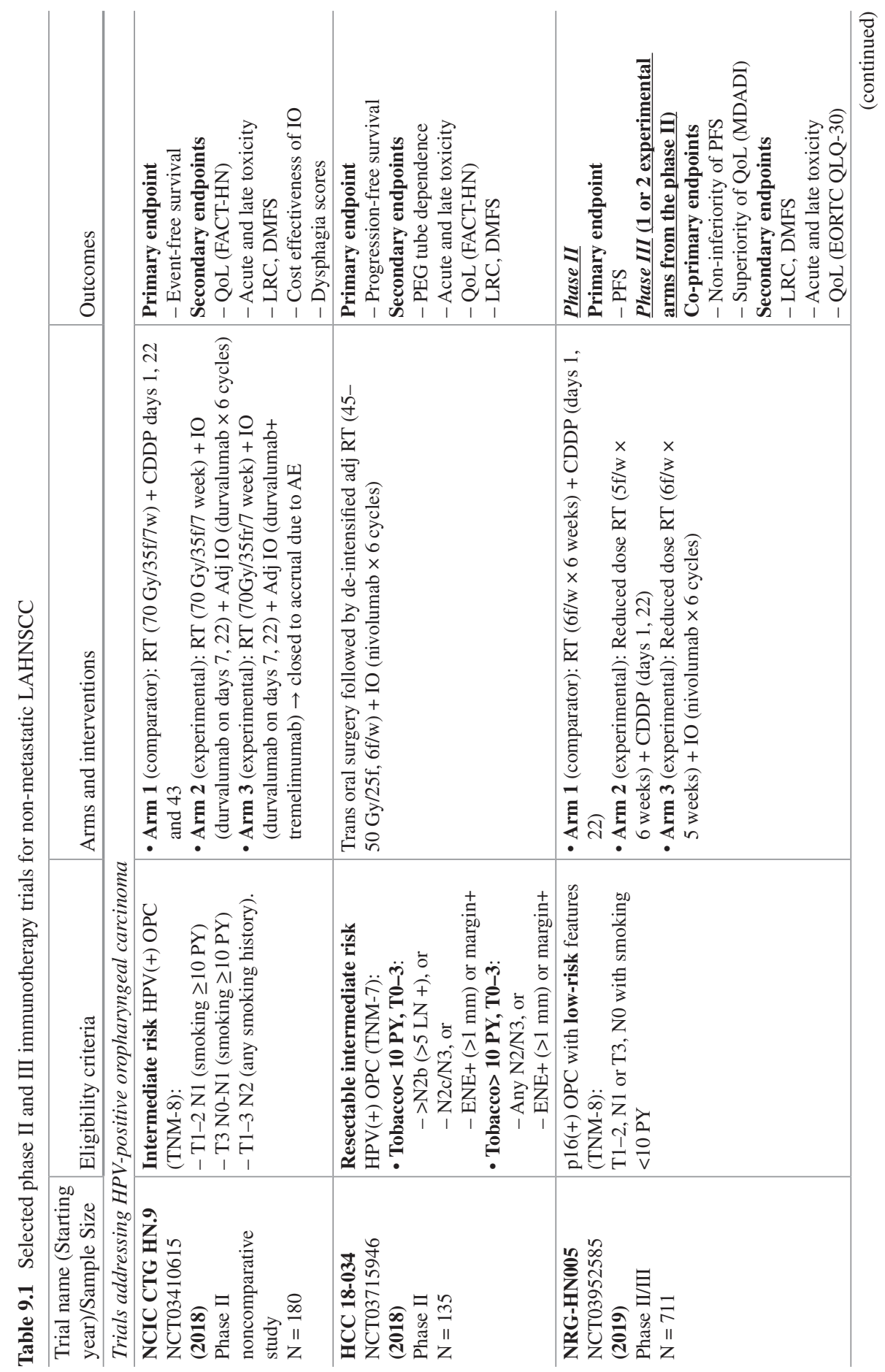




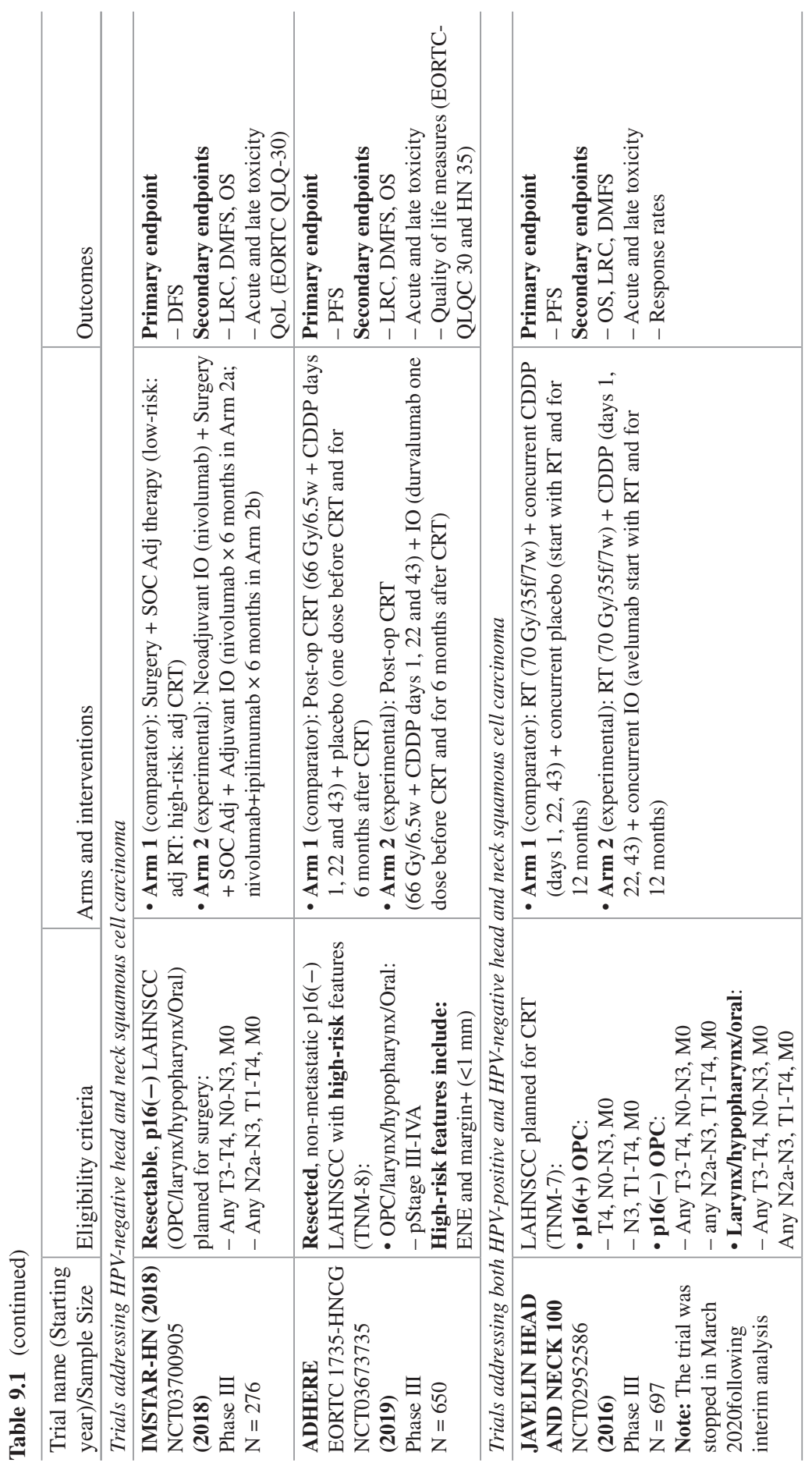




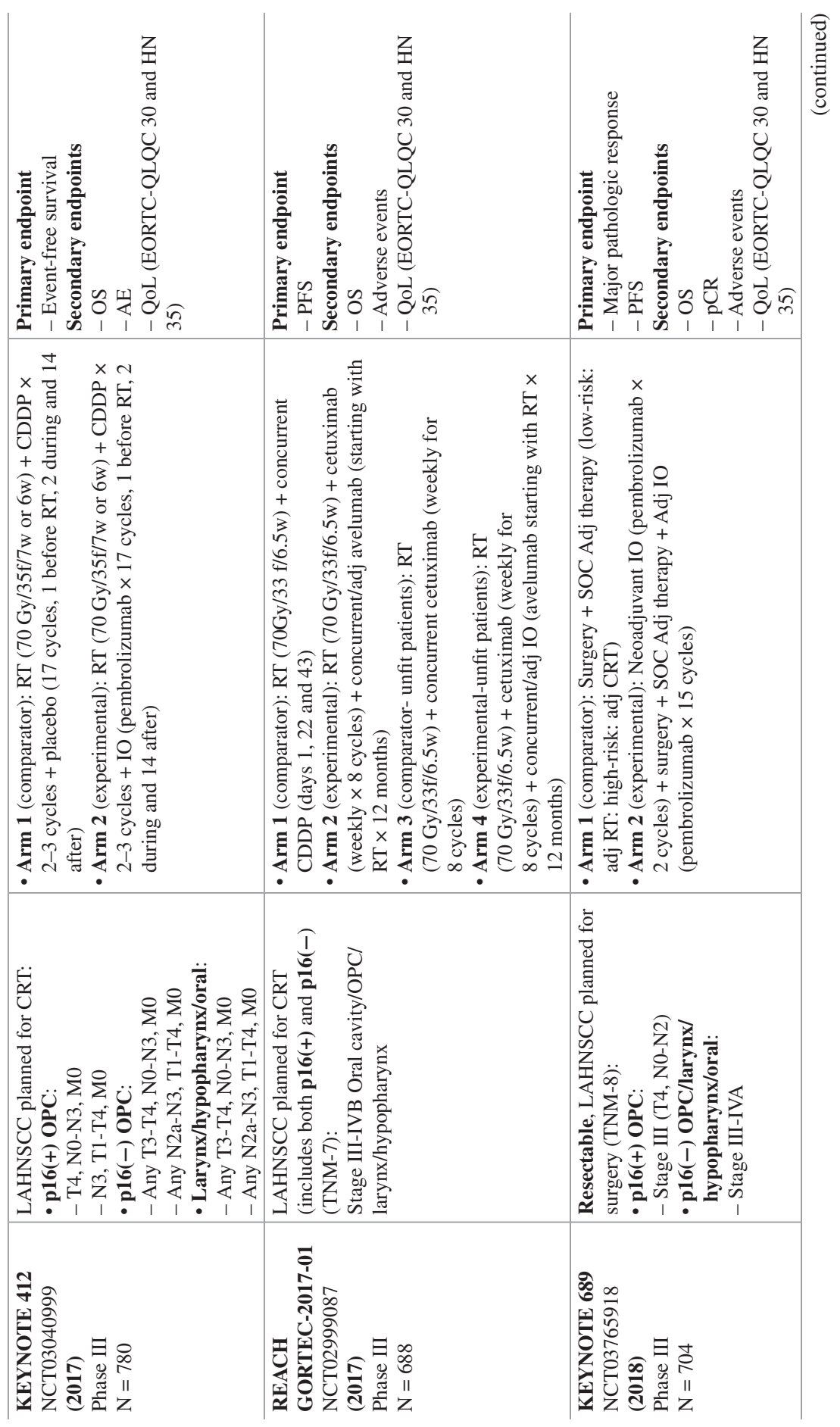




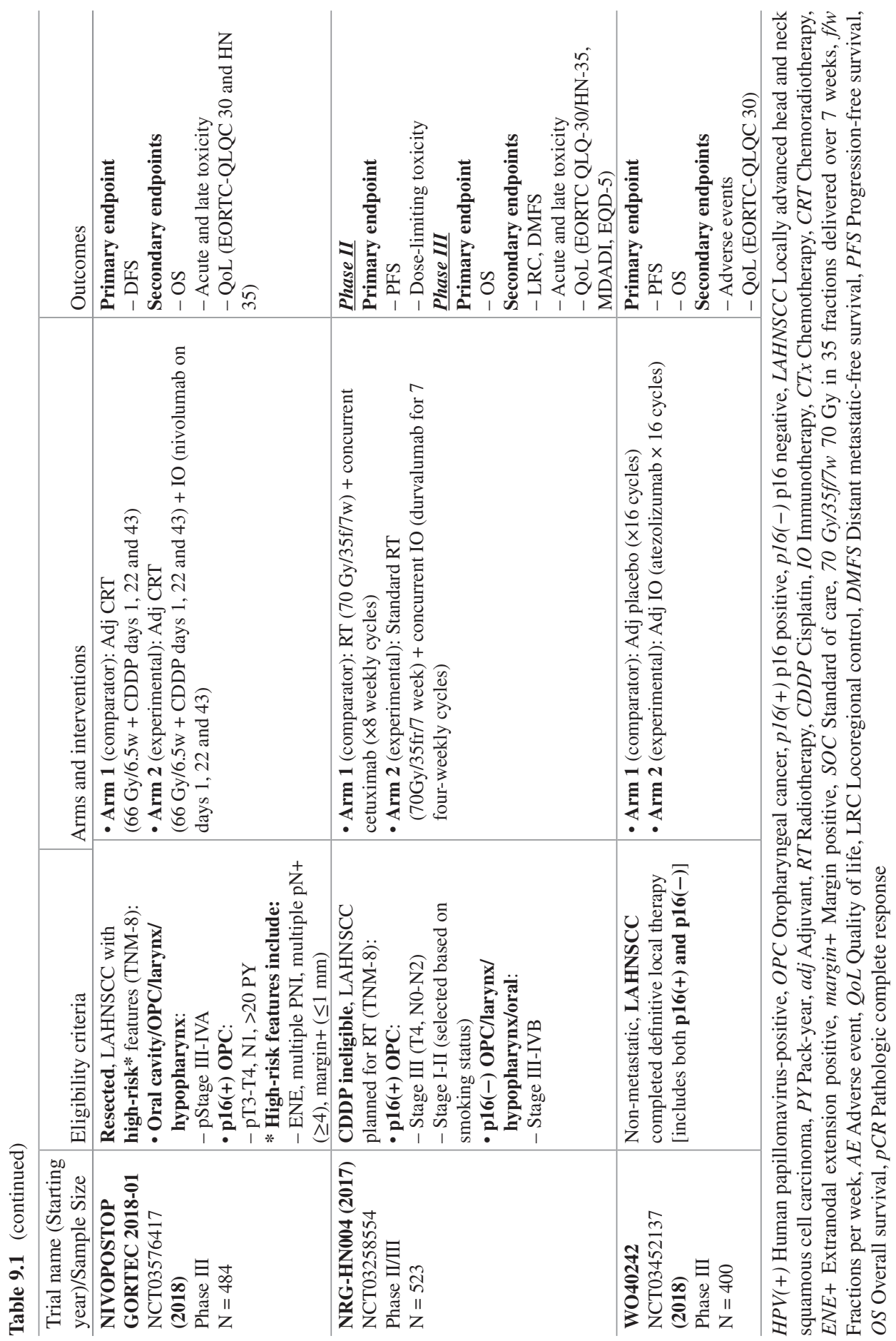


this population. Several immunotherapy trials targeting both HPV(+) (TNM-8 stage II/III) and HPV(-) LAHNSCC (TNM-8 stage III/IV) were described earlier and results are awaited. Recent genomic studies show that molecular alterations in HPV(-) LAHNSCC are common, which may provide valuable targets for immunotherapy. Another strategy is the investigation of mutated p53 [57, 58] and studies addressing novel pathways, such as Wee-1, are relevant in this regard [59, 60] as mentioned below in discussing Window of Opportunity trials.

\section{Window of Opportunity Trials Exploring Targeted Agents, Including immunotherapy}

One of the more active and potentially rewarding research areas for HPV(-) HNSCC is in the Window of Opportunity trial paradigm. Window of opportunity trials are studies where patients receive one or more new compounds between the time of cancer diagnosis and initiation of standard (mainly surgery) or investigational treatment [61]. It leverages the potentially idle time before treatment is initiated to investigate novel agents without significantly delaying the standard of care therapy [62]. Treatment response assessment can, therefore, be based on pre- and post- investigational treatment imaging and biopsy. Window of opportunity trials may, therefore, improve our understanding of pharmacodynamic parameters, and help to identify biomarkers for better patient selection. Oral cavity SCC is an ideal disease site for such trials. Several immunotherapy Window of Opportunity trials are ongoing (Table 9.1). The "WISTERIA" trial (RG_15-139, NCT03028766) [35] is evaluating the role of AZD1775 (a small molecule WEE1 inhibitor), administered before and after surgery in patients with LAHNSCC. The "SNOW-001" trial (NCT03575598) is another example in which the role of sitravatinib (a tyrosine kinases inhibitor) is evaluated combined with nivolumab administered before surgery in oral cavity SCC.

\section{Hypoxia Modification and Smoking Cessation}

Hypoxia has been identified as a contributor to radio-resistance and LRF in HNSCC $[63,64]$. Several methods have been investigated to overcome this problem [65] but without broad success [66]. For example, investigators have attempted to reduce hypoxia by blood transfusion [67] or by the administration of erythropoietin [68, 69] with RT, but disappointingly found such efforts to be not only unhelpful but apparently deleterious. Conversely, hypoxic cell radiosensitizers (e.g. nimorazole) combined with RT enhanced its effectiveness [21, 70-72] but the effect appears to be confined within the HPV(-) LAHNSCC subgroups with hypoxic tumours [21, 72]. A similar phenomenon was also observed in the TROG 0202 trial which tested the addition of tirapazamine, a hypoxic cell cytotoxin, with CCRT [73]. However, 
identifying patients with hypoxic tumours prior to RT is challenging. Various hypoxia gene signatures have been proposed although their value and availability remain to be determined [74-76] and trials addressing them have proved challenging, including tight turn-around time for the assay in different jurisdictions (especially if these are remote from the testing facility), and more recently competition with other strategies for the same patient groups (most obviously related to the recent provocative developments focusing on immunotherapy).

Perhaps, one of the most potent and available strategies to tackle tumour hypoxia is smoking cessation. Studies have shown that current smokers have the highest risk of disease recurrence and toxicity from RT compared to "never smokers" [77-80]. Evidence exists that smoking cessation could reverse blood hypoxia levels immediately to the level of "never smokers" and the LRC of such "recent quitters" appears to revert to a similar level as "never smokers" [81]. It seems imperative for radiation oncologists and health care professionals to evaluate the smoking history in HNSCC patients and promote smoking cessation strategies at the initial consultation as well as subsequently in the patient experience. The majority of current smokers appear prepared to discuss smoking cessation and accept therapy [82].

\section{Patients Unfit for Chemotherapy}

As noted, outcomes of HPV(-) LAHNSCC are unsatisfactory even with full intensity $\left(300 \mathrm{mg} / \mathrm{m}^{2}\right)$ of high dose cisplatin CCRT. Many $(>60 \%)$ are unable to receive full chemotherapy intensity $[16,83]$ or unable to tolerate chemotherapy at all due to poor general condition including organ (e.g. liver, kidney, cardiac) impairment, older age or frailty, and other comorbidities [84]. Moreover, elderly patients may not benefit from chemotherapy to the same degree [3]. Options are limited and novel approaches are needed in this under-investigated subset of LAHNSCC. Immunotherapy has emerged as a potential tool to improve outcome due to its different toxicity profile compared to traditional systemic treatments. Recently, the NRG HN-004 trial (NCT032558554) has been initiated to address this population, including both $\mathrm{HPV}(+)$ and $\mathrm{HPV}(-)$ cases. This randomized phase II/ III trial is investigating the role of durvalumab (PD-L1 inhibitor) with RT compared to cetuximab with radiation for LAHNSCC who are unable to receive cisplatin due to contraindications.

\section{Conclusion}

The landscape of LAHNSCC has changed and requires different trial questions. The disease is now generally divided into two major types: $\mathrm{HPV}(+)$ and $\mathrm{HPV}(-)$. Risk stratification (staging) and new parameters (e.g. ENE determined clinically or radiologically and pathologically) can facilitate new trial designs by enriching trial 
populations for the treatment under investigation, but also minimizing dilution of effect by exclusion of patients who are unlikely to require the intervention under study. Trials are addressing HPV(+) and HPV(-) LAHNSCC separately under the same principles of risk refinement and treatment optimization. Active research areas for non-surgical approaches include choice of RT dose/fractionation/volumes and combinations/sequences of systemic agents with radiation. Novel systemic agents, especially immunotherapy agents, are emerging but their role in the definitive treatment setting remains to be refined. Window of Opportunity trials may facilitate patient selection, identify potential therapeutic targets, and expedite drug development. A proportion of patients with LAHNSCC are unsuited for chemotherapy, such as the elderly and the frail, and may need different approaches but trials addressing these patients' needs are at a nascent phase. Efforts in this area will guide future treatment strategies in order to enhance oncologic and functional outcomes of our vulnerable head and neck cancer populations.

Disclosure Statements None.

Acknowledgments We acknowledge the Sanming Project of Medicine in Shenzhen Fund (SZSM201612024) for supporting the authors' (SHH, JL, ZX, and BOS) academic activities. We also acknowledge the O. Harold Warwick Prize of the Canadian Cancer Society for supporting the author's (BOS) academic activities.

\section{References}

1. Lydiatt W, Ridge J, Patel S, et al. Oropharynx (p16-) and hypopharynx. In: Amin M, Edge S, Greene F, et al., editors. AJCC cancer staging manual. 8th ed. New York: Springer; 2017. p. 113-21.

2. O'Sullivan B, Lydiatt W, Haughey BH, et al. HPV-mediated (p16+) oropharyngeal cancer. In: Amin M, Edge S, Greene F, et al., editors. AJCC cancer staging manual. 8th ed. New York: Springer; 2017. p. 113-21.

3. Pignon JP, le Maitre A, Maillard E, et al. Meta-analysis of chemotherapy in head and neck cancer (MACH-NC): an update on 93 randomised trials and 17,346 patients. Radiother Oncol. 2009;92:4-14.

4. Pignon JP, Bourhis J, Domenge C, et al. Chemotherapy added to locoregional treatment for head and neck squamous-cell carcinoma: three meta-analyses of updated individual data. MACH-NC Collaborative Group Meta-Analysis of Chemotherapy on Head and Neck Cancer. Lancet. 2000;355:949-55.

5. O'Sullivan B, Huang SH, Siu LL, et al. Deintensification candidate subgroups in human papillomavirus-related oropharyngeal cancer according to minimal risk of distant metastasis. J Clin Oncol. 2013;31:543-50.

6. Ang KK, Harris J, Wheeler R, et al. Human papillomavirus and survival of patients with oropharyngeal cancer. N Engl J Med. 2010;363:24-35.

7. Gillison ML, Trotti AM, Harris J, et al. Radiotherapy plus cetuximab or cisplatin in human papillomavirus-positive oropharyngeal cancer (NRG Oncology RTOG 1016): a randomised, multicentre, non-inferiority trial. Lancet. 2018;

8. Mehanna H, Robinson M, Hartley A, et al. Radiotherapy plus cisplatin or cetuximab in lowrisk human papillomavirus-positive oropharyngeal cancer (De-ESCALaTE HPV): an openlabel randomised controlled phase 3 trial. Lancet. 2018. 
9. Billfalk-Kelly A, Yu E, Su J, et al. Radiologic extranodal extension portends worse outcome in cN+ TNM-8 stage I human papillomavirus-mediated oropharyngeal cancer. Int J Radiat Oncol Biol Phys. 2019;104:1017-27.

10. Huang SH, O'Sullivan B, Su J, et al. Prognostic importance of radiologic extranodal extension in HPV-positive oropharyngeal carcinoma and its potential role in refining TNM- $8 \mathrm{cN}$ classification. Radiother Oncol. 2020;144:13-22.

11. Bonner JA, Harari PM, Giralt J, et al. Radiotherapy plus cetuximab for squamous-cell carcinoma of the head and neck. N Engl J Med. 2006;354:567-78.

12. Mirghani H, Amen F, Moreau F, et al. Oropharyngeal cancers: relationship between epidermal growth factor receptor alterations and human papillomavirus status. Eur $\mathrm{J}$ Cancer. 2014;50:1100-11.

13. O'Sullivan B, Huang SH, Perez-Ordonez B, et al. Outcomes of HPV-related oropharyngeal cancer patients treated by radiotherapy alone using altered fractionation. Radiother Oncol. 2012;103:49-56.

14. Garden AS, Fuller CD, Rosenthal DI, et al. Radiation therapy (with or without neck surgery) for phenotypic human papillomavirus-associated oropharyngeal cancer. Cancer. 2016;122:1702-7.

15. Ang KK, Zhang Q, Rosenthal DI, et al. Randomized phase III trial of concurrent accelerated radiation plus cisplatin with or without cetuximab for stage III to IV head and neck carcinoma: RTOG 0522. J Clin Oncol. 2014;32:2940-50.

16. Spreafico A, Huang SH, Xu W, et al. Impact of cisplatin dose intensity on human papillomavirus-related and -unrelated locally advanced head and neck squamous cell carcinoma. Eur J Cancer. 2016;67:174-82.

17. Yom SS, Torres-Saavedra P, Caudell JJ, et al. Reduced-Dose Radiation Therapy for HPVAssociated Oropharyngeal Carcinoma (NRG Oncology HN002). J Clin Oncol. 2021: JCO2003128.

18. Harari PM, Harris J, Kies MS, et al. Postoperative chemoradiotherapy and cetuximab for high-risk squamous cell carcinoma of the head and neck: radiation therapy oncology group RTOG-0234. J Clin Oncol. 2014;32:2486-95.

19. Bourhis J, Overgaard J, Audry H, et al. Hyperfractionated or accelerated radiotherapy in head and neck cancer: a meta-analysis. Lancet. 2006;368:843-54.

20. Lassen P, Eriksen JG, Krogdahl A, et al. The influence of HPV-associated p16-expression on accelerated fractionated radiotherapy in head and neck cancer: evaluation of the randomised DAHANCA 6\&7 trial. Radiother Oncol. 2011;100:49-55.

21. Lassen P, Eriksen JG, Hamilton-Dutoit S, et al. HPV-associated p16-expression and response to hypoxic modification of radiotherapy in head and neck cancer. Radiother Oncol. 2010;94:30-5.

22. Villaflor VM, Melotek JM, Karrison TG, et al. Response-adapted volume de-escalation (RAVD) in locally advanced head and neck cancer. Ann Oncol. 2016;27:908-13.

23. Seiwert TY, Foster CC, Blair EA, et al. OPTIMA: a phase II dose and volume de-escalation trial for human papillomavirus-positive oropharyngeal cancer. Ann Oncol. 2019;30:1673.

24. Rosenberg A, Agrawal N, Pearson A, et al. Dose and volume de-escalation for HPV-associated oropharyngeal cancer: long-term follow-up of the OPTIMA trial. J Clin Oncol. 2020;38:6575.

25. Sun Y, Li WF, Chen NY, et al. Induction chemotherapy plus concurrent chemoradiotherapy versus concurrent chemoradiotherapy alone in locoregionally advanced nasopharyngeal carcinoma: a phase 3, multicentre, randomised controlled trial. Lancet Oncol. 2016;17:1509-20.

26. Zhang Y, Chen L, Hu GQ, et al. Gemcitabine and cisplatin induction chemotherapy in nasopharyngeal carcinoma. N Engl J Med. 2019;381:1124-35.

27. Cohen EE, Karrison TG, Kocherginsky M, et al. Phase III randomized trial of induction chemotherapy in patients with N2 or N3 locally advanced head and neck cancer. J Clin Oncol. 2014;32:2735-43.

28. Haddad R, O'Neill A, Rabinowits G, et al. Induction chemotherapy followed by concurrent chemoradiotherapy (sequential chemoradiotherapy) versus concurrent chemoradiotherapy alone in locally advanced head and neck cancer (PARADIGM): a randomised phase 3 trial. Lancet Oncol. 2013;14:257-64. 
29. Marur S, Li S, Cmelak AJ, et al. E1308: phase II trial of induction chemotherapy followed by reduced-dose radiation and weekly cetuximab in patients with HPV-associated resectable squamous cell carcinoma of the oropharynx-ECOG-ACRIN cancer research group. J Clin Oncol. 2017;35:490-7.

30. Kann BH, Hicks DF, Payabvash S, et al. Multi-institutional validation of deep learning for pretreatment identification of extranodal extension in head and neck squamous cell carcinoma. J Clin Oncol. 2020;38:1304-11.

31. O'Sullivan B, Huang SH, de Almeida JR, et al. Alpha test of intelligent machine learning in staging head and neck cancer. J Clin Oncol. 2020;38:1255-7.

32. Perni S, Mohamed AS, Scott J, et al. CT-based volumetric tumor growth velocity: a novel imaging prognostic indicator in oropharyngeal cancer patients receiving radiotherapy. Oral Oncol. 2016;63:16-22.

33. Chen AM, Li J, Beckett LA, et al. Differential response rates to irradiation among patients with human papillomavirus positive and negative oropharyngeal cancer. Laryngoscope. 2013;123:152-7.

34. Lee N, Schoder H, Beattie B, et al. Strategy of using intratreatment hypoxia imaging to selectively and safely guide radiation dose de-escalation concurrent with chemotherapy for locoregionally advanced human papillomavirus-related oropharyngeal carcinoma. Int J Radiat Oncol Biol Phys. 2016;96:9-17.

35. Mazul AL, Rodriguez-Ormaza N, Taylor JM, et al. Prognostic significance of non-HPV16 genotypes in oropharyngeal squamous cell carcinoma. Oral Oncol. 2016;61:98-103.

36. Goodman MT, Saraiya M, Thompson TD, et al. Human papillomavirus genotype and oropharynx cancer survival in the United States of America. Eur J Cancer. 2015;51:2759-67.

37. Bratman SV, Bruce JP, O'Sullivan B, et al. Human papillomavirus genotype association with survival in head and neck squamous cell carcinoma. JAMA Oncol. 2016;2:823-6.

38. St Guily JL, Jacquard AC, Pretet JL, et al. Human papillomavirus genotype distribution in oropharynx and oral cavity cancer in France - the EDiTH VI study. J Clin Virol. 2011;51:100-4.

39. Nichols AC, Dhaliwal SS, Palma DA, et al. Does HPV type affect outcome in oropharyngeal cancer? J Otolaryngol Head Neck Surg. 2013;42:9.

40. Liu SZ, Zandberg DP, Schumaker LM, et al. Correlation of p16 expression and HPV type with survival in oropharyngeal squamous cell cancer. Oral Oncol. 2015;51:862-9.

41. LeConte BA, Szaniszlo P, Fennewald SM, et al. Differences in the viral genome between HPVpositive cervical and oropharyngeal cancer. PLoS One. 2018;13:e0203403.

42. Fakhry C, Blackford AL, Neuner G, et al. Association of oral human papillomavirus DNA persistence with cancer progression after primary treatment for oral cavity and oropharyngeal squamous cell carcinoma. JAMA Oncol. 2019;5:985-92.

43. Chera BS, Kumar S, Shen C, et al. Plasma circulating tumor HPV DNA for the surveillance of cancer recurrence in HPV-associated oropharyngeal cancer. J Clin Oncol. 2020;38:1050-8.

44. Wang J, Sun H, Zeng Q, et al. HPV-positive status associated with inflamed immune microenvironment and improved response to anti-PD-1 therapy in head and neck squamous cell carcinoma. Sci Rep. 2019;9:13404.

45. Andersen AS, Koldjaer Solling AS, Ovesen T, et al. The interplay between HPV and host immunity in head and neck squamous cell carcinoma. Int J Cancer. 2014;134:2755-63.

46. Lilja-Fischer JK, Eriksen JG, Georgsen JB, et al. Prognostic impact of PD-L1 in oropharyngeal cancer after primary curative radiotherapy and relation to HPV and tobacco smoking. Acta Oncol. 2020;59:666-72.

47. Zhang H, Chen J. Current status and future directions of cancer immunotherapy. J Cancer. 2018;9:1773-81.

48. Ferris RL, Blumenschein G Jr, Fayette J, et al. Nivolumab for recurrent squamous-cell carcinoma of the head and neck. N Engl J Med. 2016;375:1856-67.

49. Seiwert TY, Burtness B, Mehra R, et al. Safety and clinical activity of pembrolizumab for treatment of recurrent or metastatic squamous cell carcinoma of the head and neck (KEYNOTE-012): an open-label, multicentre, phase 1b trial. Lancet Oncol. 2016;17:956-65.

50. Burtness B, Harrington KJ, Greil R, et al. Pembrolizumab alone or with chemotherapy versus cetuximab with chemotherapy for recurrent or metastatic squamous cell carcinoma 
of the head and neck (KEYNOTE-048): a randomised, open-label, phase 3 study. Lancet. 2019;394:1915-28.

51. Cohen EEW, Soulieres D, Le Tourneau C, et al. Pembrolizumab versus methotrexate, docetaxel, or cetuximab for recurrent or metastatic head-and-neck squamous cell carcinoma (KEYNOTE-040): a randomised, open-label, phase 3 study. Lancet. 2019;393:156-67.

52. Carvalho HA, Villar RC. Radiotherapy and immune response: the systemic effects of a local treatment. Clinics (Sao Paulo). 2018;73:e557s.

53. Formenti SC, Demaria S. Combining radiotherapy and cancer immunotherapy: a paradigm shift. J Natl Cancer Inst. 2013;105:256-65.

54. Campbell AM, Decker RH: Harnessing the immunomodulatory effects of radiation therapy. Oncology (Williston Park). 2018;32:370-4, CV3.

55. Yu Y, Lee NY. JAVELIN head and neck 100: a phase III trial of avelumab and chemoradiation for locally advanced head and neck cancer. Future Oncol. 2019;15:687-94.

56. EMD Serono Inc.: EMD Serono and Pfizer Halt Phase III Head and Neck Cancer Trial. New York: Pfizer; 2020.

57. Zhou G, Liu Z, Myers JN. TP53 mutations in head and neck squamous cell carcinoma and their impact on disease progression and treatment response. J Cell Biochem. 2016;117:2682-92.

58. Wilkie MD, Anaam EA, Lau AS, et al. TP53 mutations in head and neck cancer cells determine the Warburg phenotypic switch creating metabolic vulnerabilities and therapeutic opportunities for stratified therapies. Cancer Lett. 2020;478:107-21.

59. Geenen JJJ, Schellens JHM. Molecular pathways: targeting the protein kinase Wee1 in Cancer. Clin Cancer Res. 2017;23:4540-4.

60. Bi S, Wei Q, Zhao Z, et al. Wee1 inhibitor AZD1775 effectively inhibits the malignant phenotypes of esophageal squamous cell carcinoma in vitro and in vivo. Front Pharmacol. 2019;10:864.

61. Schmitz S, Duhoux F, Machiels JP. Window of opportunity studies: do they fulfil our expectations? Cancer Treat Rev. 2016;43:50-7.

62. Farlow JL, Birkeland AC, Swiecicki PL, et al. Window of opportunity trials in head and neck cancer. J Cancer Metastasis Treat. 2019;5.

63. Nordsmark M, Overgaard J. Tumor hypoxia is independent of hemoglobin and prognostic for loco-regional tumor control after primary radiotherapy in advanced head and neck cancer. Acta Oncol. 2004;43:396-403.

64. Brizel DM, Sibley GS, Prosnitz LR, et al. Tumor hypoxia adversely affects the prognosis of carcinoma of the head and neck. Int J Radiat Oncol Biol Phys. 1997;38:285-9.

65. Dewhirst MW. A potential solution for eliminating hypoxia as a cause for radioresistance. Proc Natl Acad Sci USA. 2018;115:10548-50.

66. Brown JM, Wilson WR. Exploiting tumour hypoxia in cancer treatment. Nat Rev Cancer. 2004;4:437-47.

67. Bhide SA, Ahmed M, Rengarajan V, et al. Anemia during sequential induction chemotherapy and chemoradiation for head and neck cancer: the impact of blood transfusion on treatment outcome. Int J Radiat Oncol Biol Phys. 2009;73:391-8.

68. Overgaard J, Hoff CM, Hansen HS, et al. DAHANCA 10 - effect of darbepoetin alfa and radiotherapy in the treatment of squamous cell carcinoma of the head and neck. A multicenter, open-label, randomized, phase 3 trial by the Danish head and neck cancer group. Radiother Oncol. 2018;127:12-9.

69. Shenouda G, Zhang Q, Ang KK, et al. Long-term results of radiation therapy oncology group 9903: a randomized phase 3 trial to assess the effect of erythropoietin on local-regional control in anemic patients treated with radiation therapy for squamous cell carcinoma of the head and neck. Int J Radiat Oncol Biol Phys. 2015;91:907-15.

70. Overgaard J, Hansen HS, Overgaard M, et al. A randomized double-blind phase III study of nimorazole as a hypoxic radiosensitizer of primary radiotherapy in supraglottic larynx and pharynx carcinoma. Results of the Danish head and neck cancer study (DAHANCA) protocol 5-85. Radiother Oncol. 1998;46:135-46.

71. Overgaard J. Hypoxic modification of radiotherapy in squamous cell carcinoma of the head and neck—a systematic review and meta-analysis. Radiother Oncol. 2011;100:22-32. 
72. Toustrup K, Sorensen BS, Lassen P, et al. Gene expression classifier predicts for hypoxic modification of radiotherapy with nimorazole in squamous cell carcinomas of the head and neck. Radiother Oncol. 2012;102:122-9.

73. Rischin D, Peters LJ, O'Sullivan B, et al. Tirapazamine, cisplatin, and radiation versus cisplatin and radiation for advanced squamous cell carcinoma of the head and neck (TROG 02.02, HeadSTART): a phase III trial of the trans-Tasman radiation oncology group. J Clin Oncol. 2010;28:2989-95.

74. Deschuymer S, Sorensen BS, Dok R, et al. Prognostic value of a 15-gene hypoxia classifier in oropharyngeal cancer treated with accelerated chemoradiotherapy. Strahlenther Onkol. 2020;196:552-60.

75. Toustrup K, Sorensen BS, Metwally MA, et al. Validation of a 15-gene hypoxia classifier in head and neck cancer for prospective use in clinical trials. Acta Oncol. 2016;55:1091-8.

76. Eustace A, Mani N, Span PN, et al. A 26-gene hypoxia signature predicts benefit from hypoxia-modifying therapy in laryngeal cancer but not bladder cancer. Clin Cancer Res. 2013;19:4879-88.

77. Gillison ML, Zhang Q, Jordan R, et al. Tobacco smoking and increased risk of death and progression for patients with p16-positive and p16-negative oropharyngeal cancer. J Clin Oncol. 2012;30:2102-11.

78. Smith J, Nastasi D, Tso R, et al. The effects of continued smoking in head and neck cancer patients treated with radiotherapy: a systematic review and meta-analysis. Radiother Oncol. 2019;135:51-7.

79. Browman GP, Wong G, Hodson I, et al. Influence of cigarette smoking on the efficacy of radiation therapy in head and neck cancer. N Engl J Med. 1993;328:159-63.

80. Chen AM, Chen LM, Vaughan A, et al. Tobacco smoking during radiation therapy for headand-neck cancer is associated with unfavorable outcome. Int J Radiat Oncol Biol Phys. 2011;79:414-9.

81. Hoff CM, Grau C, Overgaard J. Effect of smoking on oxygen delivery and outcome in patients treated with radiotherapy for head and neck squamous cell carcinoma-a prospective study. Radiother Oncol. 2012;103:38-44.

82. Holland JM, Desai N, Holland E, et al. Smoking history and cessation guidance in head and neck cancer patients: a review of practice patterns at consultation. Int J Radiat Oncol Biol Phys. 2018;100:1404-5.

83. Szturz P, Wouters K, Kiyota N, et al. Low-dose vs. high-dose cisplatin: lessons learned from 59 chemoradiotherapy trials in head and neck cancer. Front Oncol. 2019;9:86.

84. Szturz P, Cristina V, Herrera Gomez RG, et al. Cisplatin eligibility issues and alternative regimens in locoregionally advanced head and neck cancer: recommendations for clinical practice. Front Oncol. 2019;9:464.

Open Access This chapter is licensed under the terms of the Creative Commons Attribution 4.0 International License (http://creativecommons.org/licenses/by/4.0/), which permits use, sharing, adaptation, distribution and reproduction in any medium or format, as long as you give appropriate credit to the original author(s) and the source, provide a link to the Creative Commons license and indicate if changes were made.

The images or other third party material in this chapter are included in the chapter's Creative Commons license, unless indicated otherwise in a credit line to the material. If material is not included in the chapter's Creative Commons license and your intended use is not permitted by statutory regulation or exceeds the permitted use, you will need to obtain permission directly from the copyright holder.

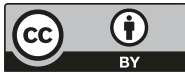

